\title{
Architectural Design of Distributed Operation Monitoring Systems
}

\author{
Chengen Wang \\ Northeaster University, Key Laboratory of Process Industry Automation \\ 3 Wenhua Street, Shenyang 110004 , China \\ wangc@mail.neu.edu.cn
}

\begin{abstract}
This paper presents an architectural design of distributed information systems used for monitoring operations of gas turbine power generation systems. The software architectural design logically uses a star topology that connects local operation monitoring systems to a central operation monitoring system. The systems are composed in accordance with the concept of three tier model on basis of COM technologies. It is argued that asynchronous and synchronous communication mechanisms are required to for different data transmission scenarios. Finally, COM+ services are implemented to support object pooling, transaction coordination and security control.
\end{abstract}

\section{Introduction}

Gas turbines are increasingly used for electrical-power generation, in addition to driving various vehicles, because of their higher energy conversion efficiencies, flexibility and lower environmental impacts. As a gas turbine based power generation system is very complex in perspectives of operation and maintenance, a power station or plant is basically incapable of independently maintaining the system without technical support from gas turbine manufacturers. Consequently, a gas turbine manufacturer is required to provide technical support throughout the product lifecycle from installation, routine operation and failure diagnosis to disposal.

To win market share and increase customer satisfaction, a gas turbine manufacturer has to quickly respond to customer's service requests. Conventionally, a gas turbine manufacturer usually dispatched service engineers to diagnose the latent troubles and fix emerging problems on installation sites, which was quite time and cost consuming. To reduce maintenance service lead-times and costs, a gas turbine manufacturer needs to exploit the benefits of information technologies. With help of distributed information systems, a gas turbine manufacturer may remotely

Please use the following format when citing this chapter:

Wang, C., 2006, in International Federation for Information

Processing, Volume 205, Research and Practical Issues of Enterprise Information Systems, eds.

Tjoa, A.M., Xu, L., Chaudhry, S., (Boston:Springer), pp.153-159. 
monitor and control the running statuses of its products which are dispersedly installed in different geographical areas.

On basis of advances in network and component technologies, researchers have studied approaches for remotely monitoring and controlling system operations. Wang et al present a framework for building web-based systems that can be used for remotely monitoring and control machines in a distributed environment [1]. The primary objectives of all maintenance strategies are to reduce equipment downtime, increase reliability and availability of the equipment which at the same time optimizes the life-cycle costs of the equipment [2]. A recent paper presents an integrated data management system for monitoring and operational evaluation of remote Renewable Energy Sources (RES) plants [3].

This paper presents architectural design of an enterprise information system for monitoring and controlling gas turbine power generation systems. The research work is triggered by a gas turbine manufacturer's need to improve service performance with constrained service capacities. It is accepted that an enterprise information system is required in order to provide operators with the best practices, to develop optimal equipment maintenance schedules, and to make best use of the company's service capacities. This paper outlines the primary considerations in the initial system design phase. The considerations concern how system components are constructed and are bound up to constitute a whole. The preliminary decisions set a conceptual system configuration and guidelines for detailed system design and development.

\section{Component Based System Architecture}

The primary objectives of implementing the operation monitoring system are multifolds. First, the system is expected to both reactively and proactively provide operators and the gas turbine manufacturer with real-time operation parameters, such as temperatures, flux, speeds, pressures and energy outputs. Second, the system should analyze and predict the equipment operation health, and develop inspection and maintenance schedules with aim of minimizing the downtime. Third, the system is responsible for generating alarming events in case that some parameters pass the set thresholds and/or some failures occur. Fourth, the system is expected to collect sufficient data and to enable the manufacturer to quickly diagnose the failure causes.

As the gas turbine manufacturer has an increasing number of customers to be supported in parallel, the operation monitoring system has to be dispersedly installed on both the manufacturer's and the customers' computers. Logically, the distributed installations of the operation monitoring systems form a star topology as shown in figure 1. A central monitoring system installed in the manufacturer is like a hub that connects local operation monitoring systems installed in the power stations.

All large applications are developed with guidance of some architecture either explicitly documented or implicitly shared in the developer's minds. Information system architectures specify the system compositions and interacting mechanisms among the constituent components. Both the central and local operation monitoring 
systems are designed in conformance with the three tier architecture, namely presentation, business and data tier.

The objective of system architectural design is to improve the information system's performance in terms of modularity, reusability, scalability, maintainability and reconfigurability. To this end, it is determined that component based system architecture be used for developing the operation monitoring systems. Among the three prevailing alternatives of CORBA, Sun J2EE and Microsoft COM, the COM technology is chosen as the software component developing tool. The COM component based architecture does not need third-party platforms. And, it is easier to implement access security mechanisms to ensure legal data access and operations on basis of authentication and authorization approaches.

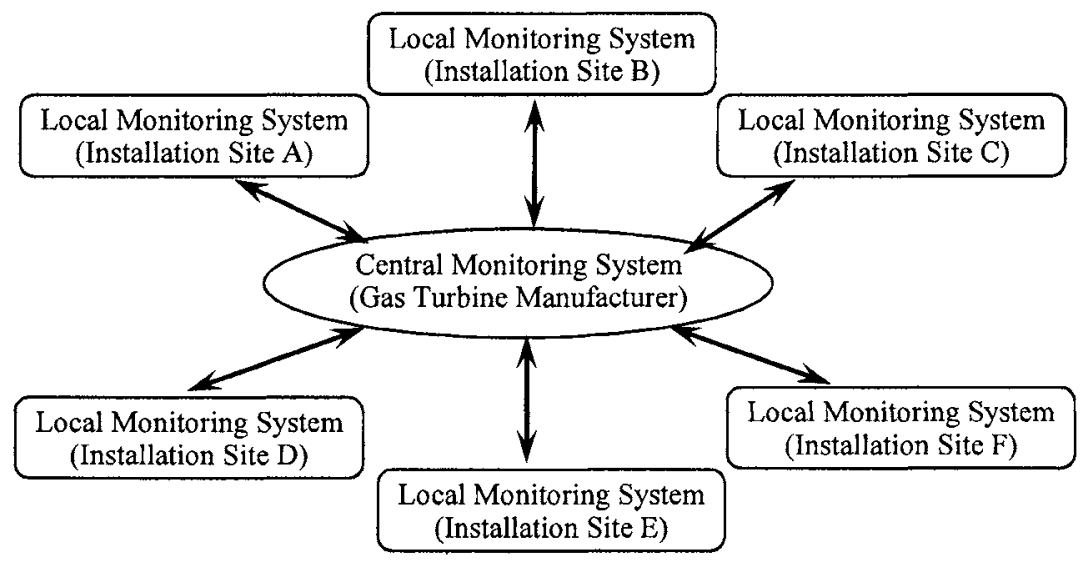

Fig. 1. Star Topology of System Installations

As shown in figure 2, the presentation tiers known as system clients are responsible for manipulating interactions between users and the business tiers through elaborately designed windows, pop-up dialogs, tabs and other view forms. The presentation tiers are to be coded as monolithic applications, which also contain some ActiveX components for graphically displaying operational data in curves, forms and diagrams. More specifically, the local operation monitoring system's clients provide operators with graphical interfaces displaying the measured operational data, and accepting user queries and commands.

The business tiers are to be coded as groups of COM components executing business procedures. A number of various sensors are embedded within the power generation system to measure real-time temperature, flux, vibration, noise, etc. COM servers at the business tier of a local operation monitoring system is responsible for retrieving data from various sensors and data collecting units by providing standard interfaces to encapsulate the measuring devices. The COM servers compare the collected data with parameter settings and analyze operational tendencies. Central monitoring system's servers contain more sophisticated fault-diagnosis algorithms, instead of simply comparing thresholds. 
The data tiers are responsible for physical storage and manipulation of data among the distributed data resources. The data tiers are to be coded as ADO components providing standard methods for database connections, data access and operations. Some business rules or operation constraints on data objects are to be enforced via means of stored procedures.

The operation monitoring system's clients and COM servers separately reside on different computers. Basically, the COM objects reactively respond to clients' requests and/or requests from other COM objects. When a client activates the servers, they reactively forward back the collected data. To reduce the communication loads between the clients and COM servers, the clients do not periodically activate the COM servers for collecting operational data. The system clients keep on waiting for event arrivals and do not activate the COM servers without human commands. For the same sake, the COM objects of the central monitoring system will not activate the COM objects of the local monitoring systems until it is requested by a service engineer.

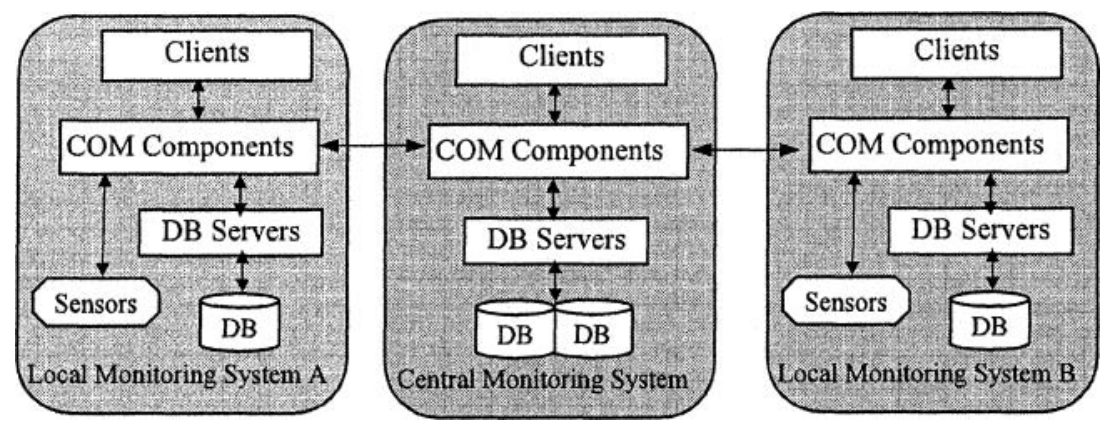

Fig. 2. Three tier System Architecture

However, when a sensor determines some abnormalities, the corresponding COM object may also proactively invoke the clients and other relevant COM objects. When one or more COM servers invoke a client, generating alarming events, the client calls embedded event handling procedures. To support the dual activations, the COM objects are designed as connectable objects implementing the IConnectionPointContainer interface containing a number of IConnectionPoint interfaces. Clients call a server's IConnectionPointContainer interface to obtain a pointer to the connectable object and call IConnectionPoint methods to create connections. Clients use the sink objects to implement the methods defined in the server's outgoing interfaces.

\section{Hybrid Communication Mechanisms}

Business requirement analysis indicates that a variety of business components are to be developed handling different business logic. For instance, a Configuration 
Control component is needed to set system parameters like types of data to be sampled, displaying modes, sampling frequencies, algorithms to be used, alarming event types and levels. Data Sampling components are developed to encapsulate sensors and compare the collected data with predefined thresholds. Data Reconciliation components check inconsistencies among a group of collected data against various thermodynamic models, filtering out noises, drifting errors, sensor bias and other inaccuracies. There are also other components responsible for fault prediction, alarming, spare part management, service supports. Instead of presenting the business components in details, this section discusses the communication mechanisms among these components.

A communication between two objects is a request and response process where a client object initiates a request and a server object responds a reply. The business nature determines that the remote monitoring system implements a hybrid mechanism of synchronous communications and asynchronous communications.

As shown in figure 3, there exist two kinds of interactions, namely vertical interactions between different tiers within a local system or the central system, and horizontal interactions between the central system's business tier and a local system's business tier. There is no communication between any two local systems. Communications between the clients at the presentation tiers and the COM objects at the business tiers are asynchronous. And, communications between COM objects of the central system's business tier and COM objects of a local system's business tier are also asynchronous. However, the asynchronous communications are implemented with two mechanisms. The clients launch asynchronous calls to COM servers in different thread spaces by implementing multi-thread models, which allows users execute multiple tasks in parallel. While COM objects of the central system and those of the local systems use message queues to transmit messages. Local COM objects use the asynchronous message queues to periodically send a gas turbine's operation parameters to the central system's COM objects. And, the central objects use the asynchronous message queues to send the local COM objects maintenance instructions, schedules, diagnosis predictions, spare parts preparations, and so on.

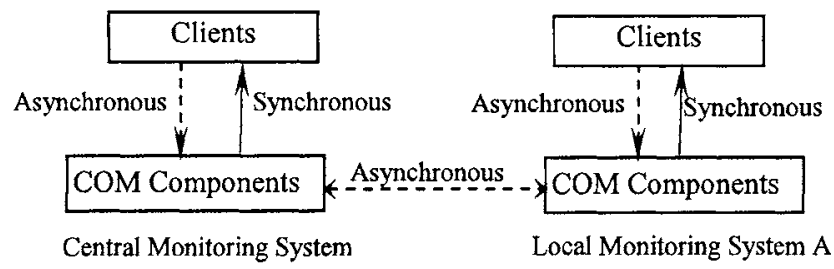

Fig. 3. Hybrid Communication Mechanisms

The asynchronous messaging mechanism does not need to create direct a connection between a sender and a receiver at the same time. The sender may put data into an existing message queue (or create a new message queue), and the receiver retrieves data from the message queue. One important reason to use the messaging mechanism is that it is more reliable and robust on unstable network connections. 
On the other hands, most communications made by COM objects in the business tier to the clients are synchronous. At the business tiers, COM objects responsible for detecting operation abnormalities contain a list of clients to be activated for emergencies. In case of urgency, the business COM objects calls the sinks' event handling methods that reside in the clients' main running processes requiring instant responses. The synchronous communication mechanism requires that the clients instantly handle the COM objects' calls before proceeding to execute other tasks.

In some cases, the central client needs to communicate with the COM objects at a local system's business tier, to handle some alarming events. As a central client is not able to directly communicate with a local COM object, the communications are implemented via a central COM object's retransmissions. The COM technology provides two approaches to support the retransmitted communications; containment and aggregation. Containment allows a COM component contains other COM components, and the outer component declares the inner components' interfaces. At run-time, the outer component delegates client's calls to the inner components and presents the outcomes to the client. Aggregation allows a COM component to expose inner components' interfaces to clients who don't know it. At run-time, the outer component passes a pointer to the inner component's interface to a client call. In our design, the containment approach is adopted to reduce communication loads and coding complexity.

\section{Interaction Mechanisms with Central Components}

As the operation monitoring systems are deployed in a star topology, some COM components at the central system's business tier are to be frequently called by a large number of dispersed clients. This gives a rise to a number of problems. First, creating and destroying a COM object for each client's call is very expensive in terms of memory, time and CPU loads. Second, some COM components are transactional, operating simultaneously on the central data sources and local data sources, which have to be strictly coordinated. Third, an effective security mechanism is required to validate numerous calls to these objects. The COM+ services are used to solve these problems.

As shown in figure 4, these COM components are added to a $\mathrm{COM}+$ server application that runs in an independent process. A COM component, integrated to a COM+ application, is known as a configured component, which is associated with a context object that defines the execution environment for the component.

$\mathrm{COM}+$ uses an object pooling technique to maintain a number of instances of a configured component in a pool, which will be activated for any call to the component. An object pool is created and maintained for each configured component. Clients are not directly served by the component's instances, but via the pool manager. Subsequently, COM+ provides transaction support to maintain the data integrity over multiple data sources. To do this, pools of the components involved in the same transaction have to be aggregated. COM+ provides role-based security framework that assigns roles to classes, interfaces, and methods. 


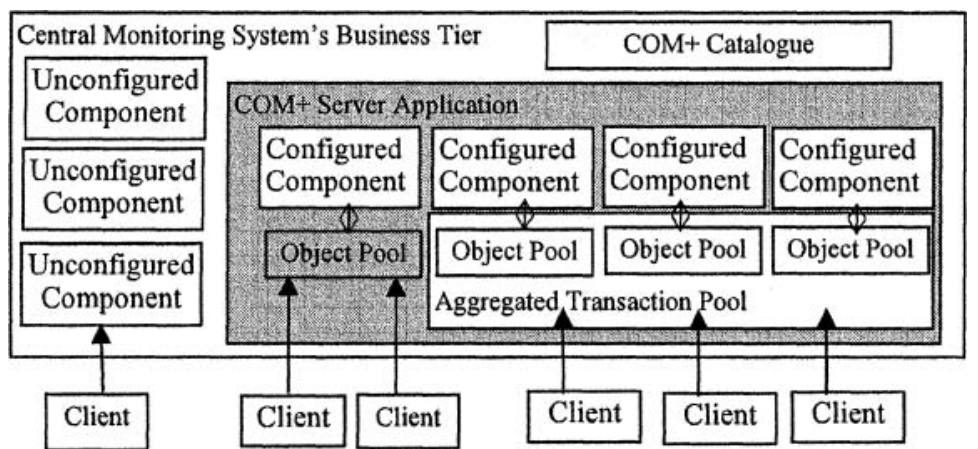

Fig. 4. Interaction Mechanisms with the Central System's COM components

\section{Conclusions}

Unique business requirements demand that operation statuses of gas turbine systems should be monitored both locally on sites and remotely over distances. Therefore, the enterprise information system is determined to consist of two kinds of loosely coupled information systems. The central operation monitoring system installed within the manufacturing company performs more sophisticated fault prediction and analysis algorithms, and develops maintenance schedules. The local operation monitoring systems installed on operation sites perform simple monitoring logic like on-line data sampling, threshold checking, fault alarming and urgency handling.

It is decided that the systems are to be conceptually designed in conformance to a three-tier architecture on basis of the COM technologies. In the distributed environments, it is identified that communications exist both within a single system, and among the central system and a local system. Asynchronous and synchronous communication mechanisms are used for different scenarios. It is further identified that $\mathrm{COM}+$ services are required to support the complicated business logic executed by the central operation monitoring system.

\section{References}

1. L. Wang, R. Sams, M. Verner, and F. Xi, Integrating Java 3D Model and Sensor Data for Remote Monitoring and Control, Robotics and Computer Integrated Manufacturing 19(1-2), 13-19 (2003).

2. S. O.T. Ogaji and R. Singh, Advanced Engine Diagnostics using Artificial Neural Networks, Applied Soft Computing 3(3), 259-271 (2003).

3. K. Papadakis, E. Koutroulis, and K. Kalaitzakis, A Server Database System for Remote Monitoring and Operational Evaluation of Renewable Energy Sources Plants, Renewable Energy 30(11), 1649-1669 (2005). 